\title{
WEAK SOLUTIONS OF A PARABOLIC-ELLIPTIC TYPE SYSTEM FOR IMAGE INPAINTING*
}

\author{
ZHENGMENG $\mathrm{JIN}^{1}$ AND XIAOPING YANG ${ }^{1}$
}

\begin{abstract}
In this paper we consider the initial boundary value problem of a parabolic-elliptic system for image inpainting, and establish the existence and uniqueness of weak solutions to the system in dimension two.
\end{abstract}

Mathematics Subject Classification. 35D05, 68U10.

Received April 5, 2009. Revised May 8, 2009.

Published online August 11, 2009.

\section{INTRODUCTION}

Image inpainting is the processing of restoring regions of missing information in digital images by using the information surrounding these regions. In the past few years, several different approaches have been proposed to tackle this complicated image processing task. One of these approaches is based on nonlinear evolution partial differential equations. The basic idea for this approach is to do a smooth propagation of the information in the region surrounding the inpainting area and interpolating level curves in a proper way $[1-3,12]$. In the pioneering work of Bertalmio et al. [2], they proposed the so called BSCB model

$$
I_{t}=\nabla^{\perp} I \cdot \nabla \triangle I
$$

for image inpainting, where $I$ is the image intensity function, $\nabla^{\perp}$ denotes perpendicular gradient $\left(-\partial_{y}, \partial_{x}\right)$. Actually (1.1) is a transport equation of the third-order that convects image Laplacian $(\triangle I)$ along isophote direction $\left(\nabla^{\perp} I\right)$.

In the subsequent work [3], a connection between the isophote direction of the image and the Navier-Stokes equation was observed and a parabolic-elliptic system was introduced to fill in the inpainting domain. The system is given by

$$
\left\{\begin{array}{l}
w_{t}+\nabla^{\perp} I \cdot \nabla w=\nu \triangle w \\
\triangle I=w
\end{array}\right.
$$

where $\nu>0$ is the viscosity coefficient. In the model (1.2), the image intensity $I$ is considered as a 'stream function' for a 2 -D incompressible flow. The Laplace of the image intensity $\Delta I$ plays the role of vorticity

\footnotetext{
Keywords and phrases. Weak solutions, parabolic-elliptic system, image inpainting.

* This work is supported by the Doctoral Programme Foundation of Education Ministry of China (N0.2003028802) and the Excellent Ph.D. Student Foundation of NUST.

${ }^{1}$ School of Science, Nanjing University of Science \& Technology, Nanjing 210094, Jiangsu, P. R. China.

jzhm353@yahoo.com.cn; yangxp@mail.njust.edu.cn
} 
of the fluid, and it is transported into the damaged region along the isophote direction $\left(\nabla^{\perp} I\right)$. Furthermore, the authors in [3] proposed to solve an anisotropic diffusion equation for $w$ in (1.2) instead of the isotropic diffusion, which is more suitable to avoid blurring of edge in the course of inpainting. It has the form:

$$
\left\{\begin{array}{l}
w_{t}+\nabla^{\perp} I \cdot \nabla w=\nu \operatorname{div}[g(|\nabla w|) \nabla w], \\
\triangle I=w .
\end{array}\right.
$$

However, the first equation for $w$ in the system (1.3) is known to be ill-posed [3,4] for a wide class of function $g$.

A different approach to image inpainting was proposed by Chan and Shen. Their work [5,6] is to minimize the TV-norm of the reconstructed images to fill in the missing data. In the later work [7,8], energy involving the curvature of the level curves is used and this is in some sense to guarantee that the level curves are connected in a smooth fashion. The equations derived from such models are highly nonlinear and of higher order.

Recently, in [14] Tai et al. proposed a two-step method to do digital image inpainting based on some geometrical considerations. In the first step, they tried to propagate the isotropic directions into the inpainting domain. Once the isotropic directions were constructed, an image was restored to fit the constructed directions in the second step. By solving the corresponding energy minimization problem in each step, they obtained a nonlinear system of PDEs.

We would like to refer [14] to the reader for further details about boundary conditions on some special situations in image inpainting. Suppose that $\Omega$ is the inpainting domain, the homogeneous Dirichlet boundary condition and the homogeneous Neumann boundary condition mean that the information surrounding the domain $\Omega$ can not be propagated into $\Omega$ through $\partial \Omega$.

Because of high nonlinearity and higher order of the equations involved, there is few theory analysis about these inpainting models. In [10], we considered the viscous BSCB equation

$$
I_{t}+\nu \triangle^{2} I=\nabla^{\perp} I \cdot \nabla \triangle I
$$

and got the existence and uniqueness of global smooth solutions of (1.4) for $\nu>0$. We also studied the vanishing viscous limits for (1.2) and obtained the existence and uniqueness of classical solutions of (1.2) for $\nu=0$. The problems we discussed in [10] were on 2-D tours, which enable us to get the results as $\nu \rightarrow 0$ in (1.2).

As a subsequent work, in this paper we consider the regularized anisotropic diffusion for $w$ in (1.3) on a 2-D bounded domain and study the following system

$$
\left\{\begin{array}{l}
w_{t}+\nabla^{\perp} I \cdot \nabla w=\nu \operatorname{div}\left[g\left(\left|\nabla G_{\sigma} * w\right|\right) \nabla w\right] \\
\triangle I=w
\end{array}\right.
$$

Here

$$
G_{\sigma}(x)=\frac{1}{4 \pi \sigma} \exp \left(-\frac{x^{2}}{4 \sigma}\right)
$$

is the Gauss kernel, the thresholding function $g$ is a non-increasing smooth function on $[0, \infty]$ satisfying the following:

- $g(0)=1, g(s)>0$;

An typical example used in applications is

$$
\text { - } \lim _{s \rightarrow \infty} \frac{\mathrm{d}^{n}}{\mathrm{~d} s^{n}} g(s)=0 \text { for each integer } n \geq 0 \text {. }
$$

$$
g(s)=\frac{1}{1+\left(\frac{s}{K}\right)^{2}},
$$

where $K>0$ is a subjective parameter.

The introducing of the regularized problem (1.5) is based on the idea of Catte et al. [4]. They pointed out in [4] that the Perona-Malik equation [13]

$$
I_{t}=\operatorname{div}[g(|\nabla I|) \nabla I] .
$$


was ill-posed due to the backward diffusion introduced in the regions where the value of $|\nabla I|$ is large for $g$ in (1.8). To make equation (1.9) well posed, they convolved $I$ with a standard mollifier inside the function $g$ to obtain the following regularized equation

$$
I_{t}=\operatorname{div}\left[g\left(\left|\nabla G_{\sigma} * I\right|\right) \nabla I\right]
$$

In [4] the existence and uniqueness of weak solutions to equation (1.10) were proved by the fixed point method. Some numerical examples were also given to indicate that the regularized model (1.10) could remove the noise effectively as the original Perona-Malik equation (1.9) during the course of image denoising.

Following the idea of [4], we discuss the regularized problem (1.5) of (1.3). Although in [3] Bertalmio et al. pointed out that the system (1.5) would be well-posed, they had not proved this fact. In this paper, we establish the existence and uniqueness of weak solutions for the system (1.5).

Observing the system (1.5), we find that the first equation in (1.5) is not a uniformly parabolic equation for $w$. It is the main difficulty in the proof of existence of weak solutions. Our method is to approximate the non-uniformly parabolic problem by a uniformly parabolic one. For the approximation problem, the Galerkin approximation method is employed to get the existence of weak solutions. Since the lower order term $\nabla^{\perp} I \cdot \nabla w$ has some special structure, which enables us to obtain the uniform estimates of $w^{\epsilon}$ in the approximate equations.

This paper is organized as follows. In Section 2 we present the initial boundary value problem of (1.5) and state the main theorem. In Section 3, we prove the existence of weak solutions to the systems (1.5) with a strictly positive diffusion. This result is used in Section 4 to establish the approximate solutions to the non-strictly positive case. In Section 4 we derive some uniform estimates for the approximate solutions which enable us to pass to the limit in the approximate system to get the existence of a weak solution as stated in Theorem 2.1.

\section{Preliminaries}

Suppose that $f: Q \rightarrow \mathbb{R}$ is a damaged image defined on a rectangle domain $Q \subset \mathbb{R}^{2}$ and $\Omega \subset \subset Q$ is the domain where the data is missing. $T$ is a positive number. We consider the initial boundary value problem for $(I, w)$ :

$$
\begin{cases}w_{t}+\nabla^{\perp} I \cdot \nabla w=\nu \operatorname{div}\left[g\left(\left|\nabla G_{\sigma} * w\right|\right) \nabla w\right], & \text { in } \Omega_{T}:=\Omega \times(0, T), \\ \triangle I=w, & \text { in } \Omega_{T}, \\ w=\triangle f, \quad I=f, & \text { on } \Gamma:=\partial \Omega \times(0, T), \\ I(x, 0)=I_{0}(x), w(x, 0)=w_{0}(x) & \text { in } \Omega .\end{cases}
$$

In (2.1) the boundary conditions ensure that filling in the information on $\Omega$ is based on the photometric information on $\partial \Omega$.

By defining $v:=w-\triangle f, u:=I-f$, problem (2.1) can be transformed into the form as follows:

$$
\begin{cases}v_{t}+\nabla^{\perp}(u+f) \cdot \nabla(v+\Delta f)=\nu \operatorname{div}\left[g\left(\left|\nabla G_{\sigma} *(v+\Delta f)\right|\right) \nabla(v+\Delta f)\right], & \text { in } \Omega_{T}, \\ \triangle u=v, & \text { in } \Omega_{T}, \\ v=0, \quad u=0, & \text { on } \Gamma, \\ u(x, 0)=u_{0}(x), \quad v(x, 0)=v_{0}(x), & \text { in } \Omega,\end{cases}
$$

where $u_{0}=I_{0}-f, v_{0}=w_{0}-\triangle f$. Throughout this paper, we study problem (2.2) for $(u, v)$ with zero-boundary value conditions.

In the following, we state our main theorem.

Theorem 2.1. Let $\Omega$ be a bounded domain of $\mathbb{R}^{2}$ with $C^{2}$ boundary. Suppose that $u_{0} \in H_{0}^{1}(\Omega) \cap W^{2, \infty}(\Omega)$, $f \in H^{3}(\Omega) \cap W^{2, \infty}(\Omega)$ with $\triangle f \in L^{\infty}(\partial \Omega)$, and $v_{0}=\triangle u_{0}$, then the initial boundary value problem (2.2) admits 
a unique pair of weak solutions $(u, v)$ such that
(a) $u \in C\left([0, T] ; H_{0}^{1}(\Omega) \cap H^{2}(\Omega)\right) \cap L^{\infty}\left(0, T ; C^{1, \alpha}(\bar{\Omega})\right)(0<\alpha<1)$,
(b) $v \in L^{2}\left(0, T ; H_{0}^{1}(\Omega)\right) \cap C\left([0, T] ; L^{2}(\Omega)\right) \cap L^{\infty}\left(\Omega_{T}\right)$,
(c) $v_{t} \in L^{2}\left(0, T ; H^{-1}(\Omega)\right)$,

which satisfies

$$
\int_{\Omega} v_{t} \varphi+\int_{\Omega}(u+f) \nabla^{\perp}(v+\triangle f) \nabla \varphi+\nu \int_{\Omega} g\left(\left|\nabla G_{\sigma} *(v+\triangle f)\right|\right) \nabla(v+\triangle f) \nabla \varphi=0
$$

and

$$
\int_{\Omega} \nabla u \nabla \varphi+\int_{\Omega} v \varphi=0
$$

for any $\varphi \in H_{0}^{1}(\Omega)$ and a.e. $t \in[0, T]$.

Note that $\nabla^{\perp}(u+f) \cdot \nabla(v+\triangle f)=-\operatorname{div}\left[(u+f) \nabla^{\perp}(v+\Delta f)\right]$. Therefore, (2.3) in Theorem 2.1 is well defined. In the following we list some lemmas that will be used in the later sections.

Lemma 2.2 ([11]). Let $E_{0}, E$ and $E_{1}$ be reflexive Banach spaces such that $E_{0} \subset E \subset E_{1}$. Suppose that the imbedding $E_{0} \rightarrow E$ is compact and the imbedding $E \rightarrow E_{1}$ is continuous. If $\left\{u_{k}\right\}$ is a bounded sequence in $L^{2}\left(0, T ; E_{0}\right)$ and $\left\{\frac{\mathrm{d} u_{k}}{\mathrm{~d} t}\right\}$ is a bounded sequence in $L^{2}\left(0, T ; E_{1}\right)$, then there exists a subsequence of $u_{k}$ which converges strongly both in $L^{2}(0, T ; E)$ and in $C\left([0, T] ; E_{1}\right)$.

Lemma $2.3([9])$. Suppose that $u \in L^{2}\left(0, T ; H_{0}^{1}(\Omega)\right)$, with $u_{t} \in L^{2}\left(0, T ; H^{-1}(\Omega)\right)$. Then $u \in C\left([0, T] ; L^{2}(\Omega)\right)$. Furthermore, the following estimate

$$
\|u\|_{C\left([0, T] ; L^{2}(\Omega)\right)} \leq C\left(\|u\|_{L^{2}\left(0, T ; H_{0}^{1}(\Omega)\right)}+\left\|u_{t}\right\|_{L^{2}\left(0, T ; H^{-1}(\Omega)\right)}\right)
$$

holds.

Lemma 2.4. Suppose that $\Omega \subset \mathbb{R}^{2}$ is a bounded domain and $u \in L^{2}(\Omega)$, then

$$
\left\|\nabla G_{\sigma} * u\right\|_{L^{\infty}(\Omega)} \leq C_{1}\|u\|_{L^{2}(\Omega)}
$$

Furthermore, if $\|u\|_{L^{2}(\Omega)}$ and $\|v\|_{L^{2}(\Omega)}$ are bounded, then

$$
\left\|g\left(\left|\nabla G_{\sigma} * u\right|\right)-g\left(\left|\nabla G_{\sigma} * v\right|\right)\right\|_{L^{\infty}(\Omega)} \leq C_{2}\|u-v\|_{L^{2}(\Omega)} .
$$

Here $C_{1}=C_{1}(\sigma), C_{2}=C_{2}\left(\sigma,\|u\|_{L^{2}(\Omega)},\|v\|_{L^{2}(\Omega)}\right)$.

Proof. By the definition of convolution, for any $x \in \Omega$, we have

$$
\nabla G_{\sigma} * u(x)=\int_{\Omega} \nabla_{x} G_{\sigma}(x-y) u(y) \mathrm{d} y
$$


Using (1.6), we deduce

$$
\begin{aligned}
\left|\nabla G_{\sigma} * u(x)\right| & \leq \frac{1}{8 \pi \sigma^{2}} \int_{\Omega} \exp \left(-\frac{(x-y)^{2}}{4 \sigma}\right)|x-y||u(y)| \mathrm{d} y \\
& \leq \frac{1}{8 \pi \sigma^{2}}\|u\|_{L^{2}(\Omega)}\left(\int_{\mathbb{R}^{2}} \exp \left(-\frac{|x-y|^{2}}{2 \sigma}\right)|x-y|^{2} \mathrm{~d} y\right)^{\frac{1}{2}} \\
& \leq C(\sigma)\|u\|_{L^{2}(\Omega)}\left(\int_{\mathbb{R}^{2}} \exp \left(-|z|^{2}\right)|z|^{2} \mathrm{~d} z\right)^{\frac{1}{2}} \\
& \leq C_{1}\|u\|_{L^{2}(\Omega)}
\end{aligned}
$$

Therefore, (2.5) holds.

On the other hand, since $g$ is smooth on $[0, \infty]$, we get

$$
\begin{aligned}
\left|g\left(\left|\nabla G_{\sigma} * u\right|\right)-g\left(\left|\nabla G_{\sigma} * v\right|\right)\right| & =\left|\int_{0}^{1} g^{\prime}\left(s\left|\nabla G_{\sigma} * u\right|+(1-s)\left|\nabla G_{\sigma} * v\right|\right)\left(\left|\nabla G_{\sigma} * u\right|-\left|\nabla G_{\sigma} * v\right|\right) \mathrm{d} s\right| \\
& \leq\left\|g^{\prime}\left(s\left|\nabla G_{\sigma} * u\right|+(1-s)\left|\nabla G_{\sigma} * v\right|\right)\right\|_{L^{\infty}(\Omega)}\left\|\nabla G_{\sigma} *(u-v)\right\|_{L^{\infty}(\Omega)} \\
& \leq C\left(\sigma,|\Omega|,\|u\|_{L^{2}(\Omega)},\|v\|_{L^{2}(\Omega)}\right)\|u-v\|_{L^{2}(\Omega)} .
\end{aligned}
$$

Therefore, (2.6) holds.

\section{EXISTENCE THEOREMS FOR STRICTLY POSITIVE DIFFUSION}

In this section we consider the following initial boundary value problem with the strictly positive diffusion:

$$
\begin{cases}v_{t}+\nabla^{\perp}(u+f) \cdot \nabla(v+\triangle f)=\nu \operatorname{div}\left[\bar{g}\left(\left|\nabla G_{\sigma} *(v+\triangle f)\right|\right) \nabla(v+\Delta f)\right], & \text { in } \Omega_{T}, \\ \triangle u=v, & \text { in } \Omega_{T}, \\ v=0, \quad u=0, & \text { on } \Gamma, \\ u(x, 0)=u_{0}(x), \quad v(x, 0)=v_{0}(x), & \text { in } \Omega,\end{cases}
$$

where we assume that $\bar{g}$ satisfies:

(i) $\bar{g}$ is a non-increasing smooth function on $[0, \infty]$ and $\bar{g}(0)=1$;

(ii) There exists a constant $A>0$ such that $A \leq \bar{g}(s) \leq 1$ for all $s \in \mathbb{R}$.

Under these assumptions we have the following theorem.

Theorem 3.1. Let $\Omega$ be a bounded domain of $\mathbb{R}^{2}$ with $C^{2}$ boundary. Suppose that $u_{0} \in H_{0}^{1}(\Omega) \cap H^{2}(\Omega)$, $f \in H^{3}(\Omega)$ and $v_{0}=\triangle u_{0}$, then the initial boundary value problem (3.1) admits a pair of weak solutions $(u, v)$ such that

which satisfies

$$
\begin{aligned}
& \text { (a) } u \in C\left([0, T] ; H_{0}^{1}(\Omega) \cap H^{2}(\Omega)\right) ; \\
& \text { (b) } v \in L^{2}\left(0, T ; H_{0}^{1}(\Omega)\right) \cap C\left([0, T] ; L^{2}(\Omega)\right) ; \\
& \text { (c) } v_{t} \in L^{2}\left(0, T ; H^{-1}(\Omega)\right)
\end{aligned}
$$

$$
\int_{\Omega} v_{t} \varphi+\int_{\Omega}(u+f) \nabla^{\perp}(v+\triangle f) \nabla \varphi+\nu \int_{\Omega} \bar{g}\left(\left|\nabla G_{\sigma} *(v+\triangle f)\right|\right) \nabla(v+\triangle f) \nabla \varphi=0
$$

and

$$
\int_{\Omega} \nabla u \nabla \varphi+\int_{\Omega} v \varphi=0
$$

for any $\varphi \in H_{0}^{1}(\Omega)$ and almost all $t \in[0, T]$. 
We point out that $u+f \in L^{\infty}\left(\Omega_{T}\right)$ since $H^{2}(\Omega) \hookrightarrow L^{\infty}(\Omega)$. Therefore, (3.2) in Theorem 3.1 is well defined.

Proof of Theorem 3.1. To prove the theorem, we apply the Galerkin approximation method. Let $\left\{\phi_{i}\right\}_{i \in \mathbb{N}}$ be the eigenfunctions of the the Laplace operator with Dirichlet boundary conditions, i.e.,

$$
-\triangle \phi=\lambda_{i} \phi_{i}, \text { in } \Omega \text { and } \phi_{i}=0 \text { on } \partial \Omega .
$$

The eigenfunctions $\phi_{i}$ are orthogonal in $H_{0}^{1}(\Omega)$ and satisfy:

$$
\left(\phi_{i}, \phi_{j}\right)_{L^{2}(\Omega)}=\delta_{i j} .
$$

Now we consider the following Galerkin ansatz for (3.1)

$$
\begin{gathered}
u^{N}(t, x)=\sum_{i=1}^{N} c_{i}^{N}(t) \phi_{i}(x), \quad v^{N}(t, x)=\sum_{i=1}^{N} d_{i}^{N}(t) \phi_{i}(x) \\
\int_{\Omega} \partial_{t} v^{N} \phi_{j}=-\nu \int_{\Omega} \bar{g}\left(\left|\nabla G_{\sigma} *\left(v^{N}+\triangle f\right)\right|\right) \nabla\left(v^{N}+\triangle f\right) \nabla \varphi_{j}-\int_{\Omega}\left(u^{N}+f\right) \nabla^{\perp}\left(v^{N}+\triangle f\right) \nabla \phi_{j}, \quad \text { for } j=1, \ldots, N \\
\int_{\Omega} \nabla u^{N} \nabla \phi_{j}=-\int_{\Omega} v^{N} \phi_{j},
\end{gathered}
$$

and

$$
v^{N}(0)=\sum_{i=1}^{N}\left(v_{0}, \phi_{i}\right)_{L^{2}(\Omega)} \phi_{i}
$$

This gives an initial value problem for a system of ODEs for $\left(d_{1}, \ldots, d_{N}\right)$ :

$$
\begin{gathered}
\partial_{t} d_{j}^{N}(t)=-\nu \int_{\Omega} \bar{g}\left(\left|\nabla G_{\sigma} *\left(\sum_{i=1}^{N} d_{i}^{N} \phi_{i}+\triangle f\right)\right|\right) \nabla\left(\sum_{i=1}^{N} d_{i}^{N} \phi_{i}+\triangle f\right) \nabla \varphi_{j} \\
-\int_{\Omega}\left(\sum_{i=1}^{N} c_{i}^{N} \phi_{i}+f\right) \nabla^{\perp}\left(\sum_{i=1}^{N} d_{i}^{N} \phi_{i}+\triangle f\right) \nabla \phi_{j}, \\
\lambda_{j} c_{j}^{N}=-d_{j}^{N}, \\
d_{j}^{N}(0)=\left(v_{0}, \phi_{j}\right)_{L^{2}(\Omega)}
\end{gathered}
$$

where $j=1, \ldots, N$. Since the right-hand side in (3.8) depends continuously on $d_{1}, \ldots, d_{N}$, according to the existence theorem of ODEs the above initial value problem has a local solution.

In the following we derive a prior bound for $v^{N}$ to ensure that the solutions of the initial value problem (3.8)-(3.10) exist globally.

At first, we get from (3.6) that

$$
\int_{\Omega} \nabla u^{N} \nabla \varphi=-\int_{\Omega} v^{N} \varphi
$$

holds for all $\varphi \in H_{0}^{1}(\Omega)$. Since $\left.u^{N}\right|_{\partial \Omega}=0$ and $\Omega$ is a bounded domain with $C^{2}$ boundary, the above equality and elliptic regularity theory imply that

$$
\left\|u^{N}(t)\right\|_{H^{2}(\Omega)} \leq C\left\|v^{N}(t)\right\|_{L^{2}(\Omega)} \text { for all } t \in[0, T],
$$


where $C$ is a constant independent of $N$. Multiplying (3.5) by $d_{j}^{N}$ and summing for $j=1, \ldots, N$, we obtain

$$
\frac{1}{2} \frac{\mathrm{d}}{\mathrm{d} t} \int_{\Omega}\left|v^{N}(t)\right|^{2}+\nu \int_{\Omega} \bar{g}\left(\left|G_{\sigma} * \nabla\left(v^{N}+\triangle f\right)\right|\right) \nabla\left(v^{N}+\triangle f\right) \nabla v^{N}=-\int_{\Omega}\left(u^{N}+f\right) \nabla^{\perp}\left(v^{N}+\triangle f\right) \nabla v^{N} .
$$

By (3.12) and assumption (ii), we conclude

$$
\frac{1}{2} \frac{\mathrm{d}}{\mathrm{d} t} \int_{\Omega}\left|v^{N}(t)\right|^{2}+A \nu \int_{\Omega}\left|\nabla v^{N}\right|^{2} \leq \nu \int_{\Omega}\left|\nabla v^{N} \nabla \triangle f\right|+\int_{\Omega}\left|f \nabla^{\perp} \triangle f \nabla v^{N}\right|+\int_{\Omega}\left|u^{N} \nabla^{\perp} \triangle f \nabla v^{N}\right| .
$$

By using Young's inequality, Sobolev imbedding theorem and (3.11), we estimate the right-hand of (3.13) to get

$$
\begin{aligned}
\nu \int_{\Omega}\left|\nabla v^{N} \nabla \triangle f\right| & \leq \frac{A \nu}{4} \int_{\Omega}\left|\nabla v^{N}\right|^{2}+C \int_{\Omega}|\nabla \triangle f|^{2} \\
\int_{\Omega}\left|f \nabla^{\perp} \triangle f \nabla v^{N}\right| & \leq \frac{A \nu}{4} \int_{\Omega}\left|\nabla v^{N}\right|^{2}+C \int_{\Omega}\left|f \nabla^{\perp} \triangle f\right|^{2} \\
& \leq \frac{A \nu}{4} \int_{\Omega}\left|\nabla v^{N}\right|^{2}+C\|f\|_{H^{3}(\Omega)}^{4}, \\
\int_{\Omega}\left|u^{N} \nabla^{\perp} \triangle f \nabla v^{N}\right| & \leq \frac{A \nu}{4} \int_{\Omega}\left|\nabla v^{N}\right|^{2}+C \int_{\Omega}\left|u^{N} \nabla^{\perp} \triangle f\right|^{2} \\
& \leq \frac{A \nu}{4} \int_{\Omega}\left|\nabla v^{N}\right|^{2}+C\left\|u^{N}(t)\right\|_{L^{\infty}(\Omega)}^{2}\|f\|_{H^{3}(\Omega)}^{2} \\
& \leq \frac{A \nu}{4} \int_{\Omega}\left|\nabla v^{N}\right|^{2}+C \int_{\Omega}\left|v^{N}(t)\right|^{2} \mathrm{~d} x\|f\|_{H^{3}(\Omega)}^{2},
\end{aligned}
$$

where the last inequality of (3.16) follows from (3.11) and $H^{2}(\Omega) \hookrightarrow L^{\infty}(\Omega)$.

Combining (3.13), (3.14), (3.15) and (3.16), we conclude

$$
\frac{1}{2} \frac{\mathrm{d}}{\mathrm{d} t} \int_{\Omega}\left|v^{N}(t)\right|^{2}+\frac{A \nu}{4} \int_{\Omega}\left|\nabla v^{N}\right|^{2} \leq C \int_{\Omega}\left|v^{N}(t)\right|^{2}+C
$$

where $C=C\left(\|f\|_{H^{3}},|\Omega|, \nu, A\right)$.

Recalling $\left.u^{N}\right|_{\partial \Omega}=0$ and integrating the above inequality from 0 to $T$, by using Gronwall's inequality and Poincaré's inequality, we conclude

$$
\begin{aligned}
\left\|v^{N}\right\|_{L^{\infty}\left((0, T) ; L^{2}(\Omega)\right)} & \leq C, \\
\left\|v^{N}\right\|_{L^{2}\left((0, T) ; H_{0}^{1}(\Omega)\right)} & \leq C,
\end{aligned}
$$

where $C=C\left(\|f\|_{H^{3}},|\Omega|, \nu, A\right)$. (3.17) implies that $\left(d_{1}^{N}, \ldots, d_{N}^{N}\right)$ are bounded and therefore a global solution to the initial value problem (3.8)-(3.10) exists. At the same time, $\left(c_{1}^{N}, \ldots, c_{N}^{N}\right)$ exist globally by (3.9). 
If we denote by $\Pi_{N}$ the projection of $L^{2}(\Omega)$ onto $\operatorname{span}\left\{\phi_{1}, \ldots, \phi_{N}\right\}$, by using assumption $(i i),(3.17),(3.18)$ and (3.11) we get

$$
\begin{aligned}
\left|\int_{\Omega_{T}} \partial_{t} v^{N} \phi\right|= & \left|\int_{\Omega_{T}} \partial_{t} v^{N} \Pi_{N} \phi\right| \\
\leq & \mid \int_{\Omega_{T}}\left[\nu \bar{g}\left(\left|\nabla G_{\sigma} *\left(v^{N}+\triangle f\right)\right|\right) \nabla\left(v^{N}+\triangle f\right) \nabla \Pi_{N} \phi \mid\right. \\
& +\left|\int_{\Omega_{T}}\left(u^{N}+f\right) \nabla^{\perp}\left(v^{N}+\triangle f\right) \nabla \Pi_{N} \phi\right| \\
\leq & C\left(\int_{\Omega_{T}}\left|\nabla\left(v^{N}+\triangle f\right)\right|^{2}\right)^{\frac{1}{2}}\left(\int_{\Omega_{T}}\left|\nabla \Pi_{N} \phi\right|^{2}\right)^{\frac{1}{2}} \\
\leq & C\|\nabla \phi\|_{L^{2}\left(\Omega_{T}\right)}
\end{aligned}
$$

for all $\phi \in L^{2}\left(0, T ; H_{0}^{1}(\Omega)\right)$. This implies

$$
\left\|\partial_{t} v^{N}\right\|_{L^{2}\left(0, T ; H^{-1}(\Omega)\right)} \leq C
$$

By using Lemma 2.3, (3.18) and (3.19), we have

$$
\left\|v^{N}\right\|_{C\left([0, T] ; L^{2}(\Omega)\right)} \leq C
$$

Using (3.18), (3.19), standard compactness results and Lemma 2.2, we obtain for a subsequence (which we still denote by $\left.v^{N}\right)$

$$
\begin{array}{rlll}
v^{N} \longrightarrow v & \text { weakly } & \text { in } L^{2}\left(0, T ; H_{0}^{1}(\Omega)\right), \\
\partial_{t} v^{N} \longrightarrow v_{t} & \text { weakly } & \text { in } L^{2}\left(0, T ; H^{-1}(\Omega)\right), \\
v^{N} \longrightarrow v & \text { strongly } & \text { in } L^{2}\left(0, T ; L^{2}(\Omega)\right), \\
v^{N}(0) \longrightarrow v_{0} & \text { strongly } & \text { in } L^{2}(\Omega) .
\end{array}
$$

By using the result that $v^{N} \longrightarrow v$ in $L^{2}\left(\Omega_{T}\right)$ and using $(2.5)$, we get

$$
\nabla G_{\sigma} *\left(v^{N}+\triangle f\right) \longrightarrow \nabla G_{\sigma} *(v+\triangle f)
$$

in $L^{2}\left(\Omega_{T}\right)^{2}$ and a.e. in $\Omega_{T}^{2}$.

It remains to show the convergence of $u^{N}$. Again using (3.11), (3.20) yields that

$$
\left\|u^{N}\right\|_{C\left([0, T] ; H^{2}(\Omega)\right)} \leq C .
$$

This implies:

$$
\begin{gathered}
u^{N} \longrightarrow u \text { weak-* in } L^{\infty}\left(\Omega_{T}\right), \\
u^{N}(t) \longrightarrow u(t) \text { weakly in } H_{0}^{1}(\Omega) \text { for all } t \in[0, T] .
\end{gathered}
$$

With the convergence properties proved so far and using assumption (ii) on $\bar{g}$, we can pass to the limits in (3.5) and (3.6) in a standard fashion (see Evans [9] for details) to get that (3.2) and (3.3) hold for $(u, v)$.

The strong convergence of $v^{N}(0) \rightarrow v_{0}$ in $L^{2}(\Omega)$ and the fact that $v \in C\left([0, T] ; L^{2}(\Omega)\right)$ give $v(0)=v_{0}$.

In the following we prove that $u(0)=u_{0}$ a.e. in $\Omega$. Recalling that $(u, v)$ satisfies

$$
\int_{\Omega} \nabla u \nabla \varphi+\int_{\Omega} v \varphi=0
$$


for any $\varphi \in H_{0}^{1}(\Omega)$ and all $t \in[0, T]$. By elliptic regularity theory we can get $u \in C\left([0, T] ; H^{2}(\Omega)\right)$, since $v \in C\left([0, T] ; L^{2}(\Omega)\right)$ and $\Omega$ is a bounded domain with $C^{2}$ boundary. Therefore, we obtain

$$
\triangle u(0)=v(0), \quad \text { a.e. in } \Omega .
$$

We have proved that $v(0)=v_{0}$ a.e. in $\Omega$. Using the condition $v_{0}=\Delta u_{0}$, we get

$$
\triangle u(0)=\triangle u_{0}, \quad \text { a.e. in } \Omega .
$$

Recalling that $u(0)=u_{0}=0$ on $\partial \Omega$, the above equality implies

$$
u(0)=u_{0}, \quad \text { a.e. in } \Omega
$$

by the Maximum Principle of Laplace equation.

\section{Existence AND Uniqueness fOR the NON-STRICTLY POSITIVE CASE}

In this section, we prove Theorem 2.1. Our method is to approximate the non-uniformly parabolic problem by an uniformly parabolic one which has a strictly positive diffusion.

We introduce a strictly positive diffusion $g_{\epsilon}$ as

$$
g_{\epsilon}=g+\epsilon
$$

With this choice of $g_{\epsilon}$, Theorem 3.1 gives the existence of the system:

$$
\begin{cases}v_{t}+\nabla^{\perp}(u+f) \cdot \nabla(v+\triangle f)=\nu \operatorname{div}\left[g_{\epsilon}\left(\left|\nabla G_{\sigma} *(v+\Delta f)\right|\right) \nabla(v+\triangle f)\right], & \text { in } \Omega_{T}, \\ \triangle u=v, & \text { in } \Omega_{T}, \\ v=0, \quad u=0, & \text { on } \Gamma, \\ u(x, 0)=u_{0}(x), \quad v(x, 0)=v_{0}(x), & \text { in } \Omega .\end{cases}
$$

We denote the solution by $\left(u_{\epsilon}, v_{\epsilon}\right)$. In the following we derive some uniform estimates of $u_{\epsilon}$ and $v_{\epsilon}$ with respect to $\epsilon$. With these estimates we can pass to the limit in the approximate system. From now on we assume $u_{0} \in H_{0}^{1}(\Omega) \cap W^{2, \infty}(\Omega), f \in H^{3}(\Omega) \cap W^{2, \infty}(\Omega)$ with $\triangle f \in L^{\infty}(\partial \Omega)$. Under this assumption, we can state the following lemma.

Lemma 4.1. The solution $v_{\epsilon} \in L^{\infty}\left(\Omega_{T}\right)$, furthermore,

$$
\left\|v_{\epsilon}\right\|_{L^{\infty}\left(\Omega_{T}\right)} \leq C
$$

where $C$ is independent of $\epsilon$.

Proof. Since

$$
u_{0} \in W^{2, \infty}(\Omega) \text { and } v_{0}=\triangle u_{0},
$$

we have $v_{0} \in L^{\infty}(\Omega)$. By $(3.2)\left(v_{\epsilon}, u_{\epsilon}\right)$ satisfies

$$
\int_{\Omega} \partial_{t} v_{\epsilon} \varphi+\int_{\Omega}\left(u_{\epsilon}+f\right) \nabla^{\perp}\left(v_{\epsilon}+\triangle f\right) \nabla \varphi+\nu \int_{\Omega} g_{\epsilon}\left(\left|\nabla G_{\sigma} *\left(v_{\epsilon}+\triangle f\right)\right|\right) \nabla\left(v_{\epsilon}+\triangle f\right) \nabla \varphi=0
$$

for any $\varphi \in H_{0}^{1}(\Omega)$ and almost all $t \in[0, T]$.

Set

$$
l=\max \left\{\|\triangle f\|_{L^{\infty}(\partial \Omega)},\left\|v_{0}+\triangle f\right\|_{L^{\infty}(\Omega)}\right\}
$$


For any $k>l$, taking $\varphi(t)=\left(v_{\epsilon}+\triangle f-k\right)_{+}(t) \in H_{0}^{1}(\Omega)$ as the test function in (4.3) and integrate from 0 to $s$, we obtain for all $s \in(0, T]$

$$
\begin{aligned}
\int_{\Omega_{s}} \partial_{t} v_{\epsilon}\left(v_{\epsilon}+\Delta f-k\right)_{+}+ & \int_{\Omega_{s}}\left(u_{\epsilon}+f\right) \nabla^{\perp}\left(v_{\epsilon}+\Delta f\right) \nabla\left(v_{\epsilon}+\triangle f-k\right) \\
& +\nu \int_{\Omega_{s}} g_{\epsilon}\left(\left|\nabla G_{\sigma} *\left(v_{\epsilon}+\triangle f\right)\right|\right) \nabla\left(v_{\epsilon}+\triangle f\right) \nabla\left(v_{\epsilon}+\triangle f-k\right)=0
\end{aligned}
$$

where $\Omega_{s}=\Omega \times(0, s)$.

Note that

$$
\begin{aligned}
\int_{\Omega_{s}} \partial_{t} v_{\epsilon}\left(v_{\epsilon}+\Delta f-k\right)_{+} & =\int_{\Omega_{s}} \partial_{t}\left(v_{\epsilon}+\Delta f-k\right)_{+}\left(v_{\epsilon}+\Delta f-k\right)_{+} \\
& =\frac{1}{2} \int_{\Omega_{s}} \frac{\mathrm{d}}{\mathrm{d} t}\left|\left(v_{\epsilon}+\Delta f-k\right)_{+}\right|^{2}
\end{aligned}
$$

and

$$
\begin{aligned}
\int_{\Omega_{s}}\left(u_{\epsilon}+f\right) \nabla^{\perp}\left(v_{\epsilon}+\Delta f\right) \nabla\left(v_{\epsilon}+\Delta f-k\right) & =\int_{S_{s}}\left(u_{\epsilon}+f\right) \nabla^{\perp}\left(v_{\epsilon}+\triangle f\right) \nabla\left(v_{\epsilon}+\Delta f-k\right) \\
& =\int_{S_{s}}\left(u_{\epsilon}+f\right) \nabla^{\perp}\left(v_{\epsilon}+\triangle f\right) \nabla\left(v_{\epsilon}+\triangle f\right)=0,
\end{aligned}
$$

where $S_{s}=\Omega_{s} \cap\left\{v_{\epsilon}+\triangle f>k\right\}$. Therefore, it follows from (4.4) that

$$
\frac{1}{2} \int_{\Omega}\left|\left(v_{\epsilon}(s)+\triangle f-k\right)_{+}\right|^{2}+\nu \int_{\Omega_{s}} g_{\epsilon}\left(\left|\nabla G_{\sigma} *\left(v_{\epsilon}+\triangle f\right)\right|\right)\left|\nabla\left(v_{\epsilon}+\triangle f-k\right)_{+}\right|^{2}=\frac{1}{2} \int_{\Omega}\left|\left(v_{0}+\triangle f-k\right)_{+}\right|^{2}=0
$$

for a.e. $s \in[0, T]$. The above equality yields that

$$
\int_{\Omega}\left|\left(v_{\epsilon}(s)+\triangle f-k\right)_{+}\right|^{2} \leq 0
$$

which implies

Let $k \rightarrow l$, we can obtain

$$
\sup _{Q_{T}}\left(v_{\epsilon}+\triangle f\right) \leq k
$$

$$
\sup _{Q_{T}}\left(v_{\epsilon}+\triangle f\right) \leq l
$$

By the similar discussion we get

$$
\inf _{Q_{T}}\left(v_{\epsilon}+\triangle f\right) \geq-l .
$$

Using (4.5), (4.6) and the fact that $\triangle f \in L^{\infty}\left(\Omega_{T}\right)$, we obtain (4.2).

Remark. Lemma 4.1 yields $\left\|v_{\epsilon}+\triangle f\right\|_{L^{\infty}\left(\Omega_{T}\right)} \leq C$. Combining (2.5), we can get

$$
\left\|\nabla G_{\sigma} *\left(v_{\epsilon}+\triangle f\right)\right\|_{L^{\infty}\left(\Omega_{T}\right)} \leq C
$$

which implies that there exists a positive constant $B$ independent of $\epsilon$ such that

$$
g_{\epsilon}\left(\left|\nabla G_{\sigma} *\left(v_{\epsilon}+\triangle f\right)\right|\right) \geq B
$$

since $g$ is a non-increasing smooth function on $[0, \infty]$ and satisfies (1.7). 
Proof of Theorem 2.1. Recalling that $\left(u^{\epsilon}, v^{\epsilon}\right)$ satisfies

$$
\int_{\Omega_{T}} \partial_{t} v_{\epsilon} \zeta+\int_{\Omega_{T}}\left(u_{\epsilon}+f\right) \nabla^{\perp}\left(v_{\epsilon}+\triangle f\right) \nabla \zeta=-\nu \int_{\Omega_{T}} g_{\epsilon}\left(\left|\nabla G_{\sigma} *\left(v_{\epsilon}+\triangle f\right)\right|\right) \nabla\left(v_{\epsilon}+\triangle f\right) \nabla \zeta
$$

for any $\zeta \in L^{2}\left(0, T ; H_{0}^{1}(\Omega)\right)$, and

$$
\int_{\Omega} \nabla u_{\epsilon} \nabla \varphi+\int_{\Omega} v_{\epsilon} \varphi=0
$$

for any $\varphi \in H_{0}^{1}(\Omega)$ and almost all $t \in[0, T]$.

(4.7) shows that the approximate systems are uniform and strictly positive diffusion for $v_{\epsilon}$. Therefore, taking $\zeta=v_{\epsilon}$ in (4.8) and $\varphi=u_{\epsilon}(t)$ in (4.9) respectively as the test functions, using the similar discussion as in the proof of Theorem 3.1, we obtain

$$
\begin{aligned}
\left\|v_{\epsilon}\right\|_{L^{2}\left(0, T ; H_{0}^{1}(\Omega)\right)} & \leq C \\
\left\|\partial_{t} v_{\epsilon}\right\|_{L^{2}\left(0, T ; H^{-1}(\Omega)\right)} & \leq C \\
\left\|v_{\epsilon}\right\|_{C\left([0, T] ; L^{2}(\Omega)\right)} & \leq C \\
\left\|u_{\epsilon}\right\|_{C\left([0, T] ; H^{2}(\Omega)\right)} & \leq C .
\end{aligned}
$$

Recalling (4.2), by using standard compactness properties and Lemma 2.2, we obtain the convergence

$$
\begin{gathered}
v_{\epsilon} \longrightarrow v \quad \text { weakly in } L^{2}\left(0, T ; H_{0}^{1}(\Omega)\right), \\
\partial_{t} v_{\epsilon} \longrightarrow v_{t} \quad \text { weakly in } L^{2}\left(0, T ; H^{-1}(\Omega)\right), \\
v_{\epsilon} \longrightarrow v \quad \text { strongly in } L^{2}\left(0, T ; L^{2}(\Omega)\right), \\
v_{\epsilon} \longrightarrow v \quad \text { weak-* } \text { in } L^{\infty}\left(\Omega_{T}\right), \\
u_{\epsilon} \longrightarrow u \quad \text { weak-* in } L^{\infty}\left(\Omega_{T}\right), \\
u_{\epsilon}(t) \longrightarrow u_{(t)} \text { weakly in } H_{0}^{1}(\Omega) \text { for all } t \in[0, T] .
\end{gathered}
$$

It remains to show that $(u, v)$ fulfills $(2.3)-(2.4)$ in Theorem 2.1. With these convergence (4.10), (4.11) and (4.13), it is obvious that

$$
\int_{\Omega_{T}} \partial_{t} v_{\epsilon} \zeta+\int_{\Omega_{T}}\left(u_{\epsilon}+f\right) \nabla^{\perp}\left(v_{\epsilon}+\Delta f\right) \nabla \zeta \longrightarrow \int_{\Omega_{T}} \partial_{t} v \zeta+\int_{\Omega_{T}}(u+f) \nabla^{\perp}(v+\triangle f) \nabla \zeta
$$

for any $\zeta \in L^{2}\left(0, T ; H_{0}^{1}(\Omega)\right)$. Recalling that $v_{\epsilon} \longrightarrow v$ in $L^{2}\left(\Omega_{T}\right)$ and using $(2.5)$, we get

$$
\nabla G_{\sigma} *\left(v_{\epsilon}+\triangle f\right) \longrightarrow \nabla G_{\sigma} *(v+\triangle f)
$$

in $L^{2}\left(\Omega_{T}\right)^{2}$ and a.e. in $\Omega_{T}{ }^{2}$. Note that $g_{\epsilon} \longrightarrow g$ uniformly, therefore,

$$
g_{\epsilon}\left(\left|\nabla G_{\sigma} *\left(v_{\epsilon}+\triangle f\right)\right|\right) \longrightarrow g\left(\left|\nabla G_{\sigma} *(v+\triangle f)\right|\right) \quad \text { a.e in } \Omega_{T} .
$$


Since $0<g_{\epsilon} \leq C$ for small $\epsilon$, by using Lebesgue dominated convergence theorem, (4.15) and (4.10), we get

$$
\nu \int_{\Omega_{T}} g_{\epsilon}\left(\left|\nabla G_{\sigma} *\left(v_{\epsilon}+\triangle f\right)\right|\right) \nabla\left(v_{\epsilon}+\triangle f\right) \nabla \zeta \longrightarrow \nu \int_{\Omega_{T}} g\left(\left|\nabla G_{\sigma} *(v+\triangle f)\right|\right) \nabla(v+\triangle f) \nabla \zeta
$$

for any $\zeta \in L^{2}\left(0, T ; H_{0}^{1}(\Omega)\right)$.

Therefore, letting $\epsilon \rightarrow 0$ in (4.8), by the arbitrary of $\zeta \in L^{2}\left(0, T ; H_{0}^{1}(\Omega)\right)$, we obtain that (2.3) holds for any $\varphi \in H_{0}^{1}(\Omega)$ and a.e. $t \in[0, T]$. At the same time, letting $\epsilon \rightarrow 0$ in (4.9), using (4.12) and (4.14), we get that (2.4) holds for any $\varphi \in H_{0}^{1}(\Omega)$ and a.e. $t \in[0, T]$.

The facts that $u(0)=u_{0}, v(0)=v_{0}$ and $u \in C\left([0, T] ; H_{0}^{1}(\Omega) \cap H^{2}(\Omega)\right)$ follow the similar discussion as in the proof of Theorem 3.1. Furthermore, since $(u, v)$ satisfies $(2.4)$ and $v \in L^{\infty}\left(\Omega_{T}\right)$, the Schauder estimates of elliptic equations imply $u \in L^{\infty}\left(0, T ; C^{1, \alpha}(\bar{\Omega})\right)(0<\alpha<1)$. This proves the existence of $(u, v)$ to problem $(2.2)$.

In the following we prove the uniqueness. Suppose that $\left(u_{1}, v_{1}\right)$ and $\left(u_{2}, v_{2}\right)$ are two pairs of weak solutions to problem (2.2). By defining

$$
\beta_{i}(t)=g\left(\left|\nabla G_{\sigma} * v_{i}(t)\right|\right), \quad i=1,2,
$$

we have

$$
\int_{\Omega} \partial_{t}\left(w_{1}-w_{2}\right) \varphi+\int_{\Omega}\left(I_{1} \nabla^{\perp} w_{1}-I_{2} \nabla^{\perp} w_{2}\right) \nabla \varphi+\nu \int_{\Omega}\left(\beta_{1}(t) \nabla w_{1}-\beta_{2}(t) \nabla w_{2}\right) \nabla \varphi=0
$$

for any $\varphi \in H_{0}^{1}(\Omega)$ and a.e. $t \in[0, T]$. Here

$$
w_{i}=v_{i}+\triangle f, \quad I_{i}=u_{i}+f, \quad i=1,2 .
$$

In (4.16) taking $\varphi=\left(w_{1}-w_{2}\right)(t)$ as the test function, we obtain

$$
\begin{array}{r}
\frac{1}{2} \frac{\mathrm{d}}{\mathrm{d} t} \int_{\Omega}\left|\left(v_{1}-v_{2}\right)(t)\right|^{2}+\nu \int_{\Omega} \beta_{1}(t)\left|\nabla\left(v_{1}-v_{2}\right)\right|^{2}=\nu \int_{\Omega}\left[\left(\beta_{2}-\beta_{1}\right)(t)\right] \nabla\left(v_{2}+\triangle f\right) \nabla\left(v_{1}-v_{2}\right) \\
+\nu \int_{\Omega}\left(u_{1}-u_{2}\right) \nabla^{\perp}\left(v_{2}+\triangle f\right) \nabla\left(v_{1}-v_{2}\right) .
\end{array}
$$

Note that

and

$$
\beta_{1}(t)=g\left(\left|\nabla G_{\sigma} * v_{1}(t)\right|\right) \geq B>0
$$

$$
\left\|\left(\beta_{1}-\beta_{2}\right)(t)\right\|_{L^{\infty}(\Omega)} \leq C\left\|\left(v_{1}-v_{2}\right)(t)\right\|_{L^{2}(\Omega)}
$$

by (2.6). Combining (4.17), (4.18), (4.19) and using Hölder's inequality, we obtain

$\frac{1}{2} \frac{\mathrm{d}}{\mathrm{d} t}\left\|\left(v_{1}-v_{2}\right)(t)\right\|_{L^{2}(\Omega)}^{2}+B \nu\left\|\nabla\left(v_{1}-v_{2}\right)(t)\right\|_{L^{2}(\Omega)}^{2} \leq C\left\|\nabla\left(v_{2}+\triangle f\right)\right\|_{L^{2}(\Omega)}\left\|\nabla\left(v_{1}-v_{2}\right)(t)\right\|_{L^{2}(\Omega)}\left\|\left(v_{1}-v_{2}\right)(t)\right\|_{L^{2}(\Omega)}$,

where we used the fact that

$$
\left\|\left(u_{1}-u_{2}\right)(t)\right\|_{L^{\infty}(\Omega)} \leq C\left\|\left(v_{1}-v_{2}\right)(t)\right\|_{L^{2}(\Omega)}
$$

for all $t \in[0, T]$. Using Young's inequality for the last term in (4.20), we get

$$
\frac{1}{2} \frac{\mathrm{d}}{\mathrm{d} t}\left\|\left(v_{1}-v_{2}\right)(t)\right\|_{L^{2}(\Omega)}^{2} \leq C\left\|\nabla\left(v_{2}+\Delta f\right)\right\|_{L^{2}(\Omega)}^{2}\left\|\left(v_{1}-v_{2}\right)(t)\right\|_{L^{2}(\Omega)}^{2} .
$$

Recalling that $v_{1}(0)=v_{2}(0)=v_{0}$ and $v_{2}+\triangle f \in L^{2}\left(0, T ; H^{1}(\Omega)\right)$, by using Gronwall's inequality, (4.22) implies

$$
v_{1}=v_{2} \quad \text { a.e. in } \Omega_{T},
$$

which implies

$$
u_{1}=u_{2} \quad \text { a.e. in } \Omega_{T}
$$

by (4.21). This completes the proof of Theorem 2.1. 


\section{REFERENCES}

[1] C. Ballester, M. Bertalmio, V. Caselles, G. Sapiro and J. Verdera, Filling-in by joint interpolation of vector fields and gray levels. IEEE Trans. Image Process. 10 (2001) 1200-1211.

[2] M. Bertalmio, G. Sapiro, V. Caselles and C. Ballsester, Image Inpainting. Computer Graphics, SIGGRAPH (2000) $417-424$.

[3] M. Bertalmio, A. Bertozzi and G. Sapiro, Navier-Stokes, fluid-dynamics and image and video inpainting, in Proc. Conf. Comp. Vision Pattern Rec. (2001) 355-362.

[4] F. Catte, P.L. Lions, J.M. Morel and T. Coll, Image selective smoothing and edge detection by nonlinear diffusion. SIAM. J. Num. Anal. 29 (1992) 182-193.

[5] T. Chan and J. Shen, Variational restoration of nonflat image feature: Models and algorithms. SIAM J. Appl. Math. 61 (2000) $1338-1361$.

[6] T. Chan and J. Shen, Mathematical models for local nontexture inpaintings. SIAM J. Appl. Math. 63 (2002) $1019-1043$.

[7] T. Chan, S. Kang and J. Shen, Euler's elastica and curvature based inpaintings. SIAM J. Appl. Math. 63 (2002) 564-592.

[8] T. Chan, J. Shen and L. Vese, Variational PDE models in image processing. Notices Am. Math. Soc. 50 (2003) 14-26.

[9] L.C. Evans, Partial differental equations. American Mathematical Society (1998).

[10] Z.M. Jin and X.P. Yang, Viscosity analysis on the BSCB models for image inpainting. Chinese Annals of Math. (Ser. A) (to appear).

[11] J.L. Lions, Quelques méthodes de résolution des problèmes aux limites non linéaries. Dunod (1969).

[12] S. Masnou, Disocclusion: a variational approach using level lines. IEEE Trans. Image Process. 11 (2002) 68-76.

[13] P. Perona and J. Malik, Scale-space and edge detection using anisotropic diffusion. IEEE Trans. Pattern Anal. Machine Intell. 12 (1990) 629-639.

[14] X.C. Tai, S. Osher and R. Holm, Image inpainting using a TV-Stokes equation, in Image Processing based on partial differential equations, X.C. Tai, K.-A. Lie, T.F. Chan and S. Osher Eds., Springer, Heidelberg (2007) 3-22. 